Conference abstract POT02

\title{
Development of Intermediate Layer with Improved Diffusional Barrier
}

\author{
M. T. JAKLIČ, R. JUREČIČ \\ Lek Pharmaceuticals,d.d., Sandoz Development Center Slovenia, Verovškova 57, 1526 Ljubljana, Slovenia \\ E-mail: miha.jaklic@sandoz.com (M. T. Jaklič), rok.jurecic@sandoz.com (R. Jurečič)
}

Sci Pharm. 2010; 78: 717

doi:10.3797/scipharm.cespt.8.POT02

Delayed release dosage form development usually requires an effective intermediate layer for separation of acid liable drug and acidic gastric polymer. A case is presented where an effective intermediate layer was developed for duloxetine pellets. Duloxetine pellets were produced in Wurster process using suspension/solution layering technique. Neutral pellet cores were coated with duloxetine layer, intermediate layer and gastric layer. Intermediate layer usually comprises water soluble polymer (e. g. povidone, hypromellose); additionally talc may be added as filler and a processing aid (it reduces tackiness of coating dispersion). These kind of intermediate layer compositions provide simple separation of API and gastric layer and are usually sufficient for effective separation. In duloxetine pellets development, however, accelerated stability tests indicated poor long term stability. Diffusion of acidic species (e. g. phthalic acid is a hypromellose phthalate degradation product) across intermediate layer was suspected. Addition of small amounts of sodium chloride (4.8\% wt./wt. relative to mass of intermediate layer, dissolved in aqueous coating dispersion) drastically improved stability of duloxetine pellets. Increase in coat thickness resulted in further decrease of duloxetine degradation products: this fact indicates that stabilization is induced by strengthening the diffusion barrier and thus minimizing diffusion of acidic species between the layers. Scanning electron microscope images were used to determine the structure of intermediate coat: talc particles are bonded with hypromellose: within hypromellose phase small crystals of sodium chloride (sub micrometer size range) were detected. Sodium chloride is dissolved in coating dispersion and once applied onto pellet surface it re-crystallizes in form of small crystals (due to fast water removal during coating). It is sugested that these finely dispersed sodium chloride crystals within hypromellose phase provide effective diffusional barrier which prevents migration of acidic species between layers and thus enabling stabilization of duloxetine. In order to assure long term stability of duloxetine pellets it was shown that an effective diffusional barrier is required. 